\title{
Components for the LISA local interferometry
}

\author{
Gerhard Heinzel, Frank Steier, Roland Fleddermann, \\ Benjamin Sheard, Karsten Danzmann \\ Max-Planck-Institut für Gravitationsphysik (Albert-Einstein-Inst.) Hannover, \\ Callinstrasse 38, D-30167 Hannover, Germany
}

\begin{abstract}
This article describes some preliminary results on essential components for the LISA interferometry, namely photodiode preamplifiers and voltage references.
\end{abstract}

Keywords: LISA, interferometry, photodiodes, voltage references

PACS: $04.80 . \mathrm{Nn}, 07.60 . \mathrm{Ly}, 42.25 . \mathrm{Hz}, 95.55 . \mathrm{Ym}$

\section{INTRODUCTION}

The basic measurement in LISA is the distance fluctuation between two free-floating test masses aboard drag-free spacecraft separated by approximately 5 million $\mathrm{km}$. Six such measurements are continuously done along the three arms of the triangular constellation of three identical spacecraft. Complications arise because due to orbital mechanics [1] the armlengths change by about $\pm 1 \%$ on timescales of the order of one year, consequently the angles in the triangle change by about $\pm 1.5^{\circ}$, and the spacecraft have a velocity relative to each other of about $\pm 15 \mathrm{~m} / \mathrm{s}$ along the line of sight which causes a Doppler shift of about $\pm 15 \mathrm{MHz}$ in the light beams going back and forth.

Separation of the measurement into three parts (see Figure 1) allows simplified development, testing and integration of subsystems by separately tackling two challenging tasks: (a) measurement of the free-floating test mass with all its alignment issues, coupling of jitter etc. and (b) interferometry along the 5 million $\mathrm{km}$ arms with its own set of challenges.

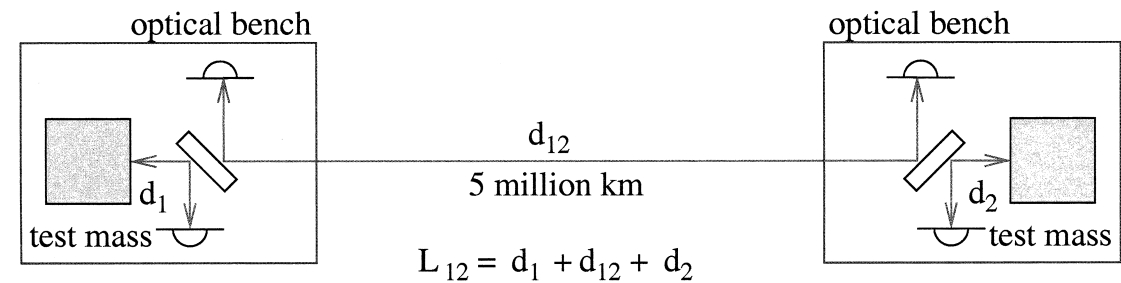

FIGURE 1. Concept of 'split interferometry'

\section{OPTICAL BENCH}

Figure 2 shows a conceptual overview of one optical bench and a possible implementation. More details can be found in [2, 3, 4].

CP873, Laser Interferometer Space Antenna-6 ${ }^{\text {th }}$ International LISA Symposium, edited by S. M. Merkowitz and J. C. Livas

(C) 2006 American Institute of Physics 978-0-7354-0372-7/06/\$23.00 


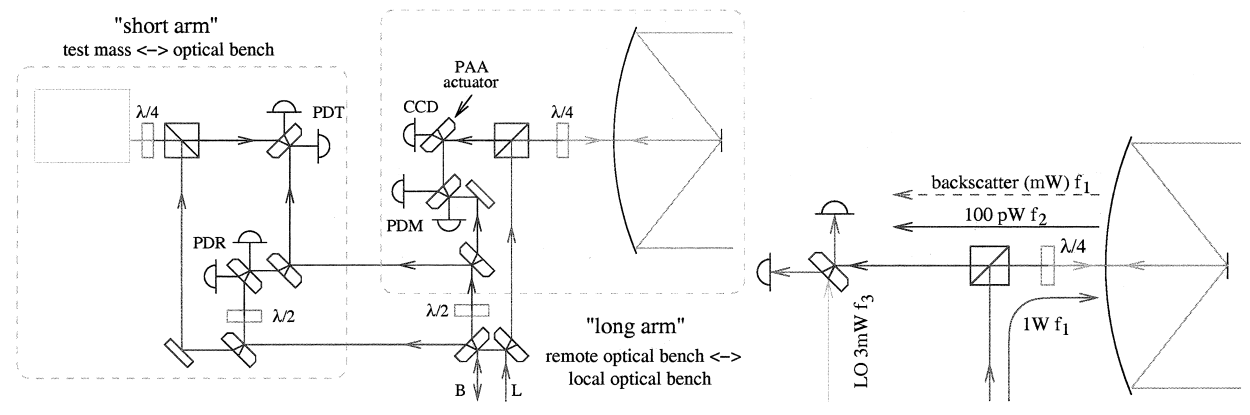

FIGURE 2. Optical bench

One new feature of the optical layout is the so-called 'frequency swapping', illustrated in the right part of Figure 2. Old layouts used the outgoing laser $\left(f_{1}\right)$ also as local oscillator (LO) for the incoming light. Any backscatter would thus add to the LO with uncontrolled phase. Frequency swapping uses a different frequency $\left(f_{3}\right)$ as LO, such that the signal at $\left|f_{2}-f_{3}\right|$ is at a different frequency from the backscatter beat $\left|f_{1}-f_{3}\right|$. The different frequency can conveniently be obtained from the second laser (mainly serving the second optical bench).

The LO light is exchanged between the two optical benches with one single-mode PM fiber used bidirectionally in the same polarization state ('B' in Figure 2). The phase shift in both directions must be the same to $\mu \mathrm{rad}(\mathrm{pm})$ level. Laboratory experiments to verify this assumption are under way.

\section{PHOTODIODE PREAMPLIFIERS}

On each optical bench there will be at least 2 quadrant photodiodes (PDM and PDT in Figure 2) and one single-element diode (PDR). For redundancy, this number will be doubled, and PDR may also be a quadrant diode, such that we have around 20 photodiode channels to be processed. Each of them will need to record a beatnote in the range of $\approx 3 \ldots 20 \mathrm{MHz}$ with high phase fidelity[5]. The signal-to-noise ratio is most critical in PDM where the beatnote resulting from the weak incoming light must be detected, and hence the requirements for PDM will be taken as guideline here.

Assuming a power of the signal beam $P_{\text {sig }}$ of $10 \mathrm{pW}$ per channel (the incoming $\approx 100 \mathrm{pW}$ being split in 8 channels), a photodiode efficiency of $\eta=0.7 \mathrm{~A} / \mathrm{W}$ and a local oscillator power $P_{\text {LO }}$ of $100 \mu \mathrm{W}$ per quadrant $\left(P_{\mathrm{LO}} \gg P_{\text {sig }}\right)$, we can compute the $\mathrm{DC}$, signal and noise components in the photocurrent as follows:

$$
\begin{aligned}
I_{\mathrm{DC}} & =\eta P_{\mathrm{LO}}=70 \mu \mathrm{A} \\
\widetilde{I_{\text {sig }}} & =2 \eta \sqrt{P_{\mathrm{LO}} P_{\mathrm{sig}}} \cos \omega t=44 \mathrm{nA}_{\mathrm{pk}}, \\
\widetilde{I_{\text {shot }}} & =\sqrt{2 e \eta P_{\mathrm{LO}}}=4.7 \mathrm{pA} / \sqrt{\mathrm{Hz}} .
\end{aligned}
$$

This is the base for deriving requirements on the photodiode and its preamplifier. 
Due to the high signal frequencies and low signal levels it is assumed that the preamplifiers must be right next to the photodiodes, i.e. on the optical bench, such that their power dissipation and its stability are also important. The DC photocurrent scales linearly with the local oscillator power $P_{\text {LO }}$, while both the signal and the shot noise are proportional to its square root, such that the shot-noise limited signal-to-noise ratio is, as expected, independent of $P_{\mathrm{LO}}$ for $P_{\mathrm{LO}} \gg P_{\text {sig. }}$. The electronic noise of the photodiodepreamplifier combination should ideally be significantly lower than the shot noise, e.g. $\widetilde{I_{\mathrm{el}}} \lesssim 1 \mathrm{pA} / \sqrt{\mathrm{Hz}}$.

We have begun to study design options for the preamplifier. In the left part of Figure 3, the photocurrent is converted into a voltage by a resistor and then amplified. If the resistor is $50 \ldots 100 \Omega$, the signal could be transmitted to a remote preamplifier through a coaxial cable. Unfortunately, however, the current noise of a $50 \Omega$ resistor is $18 \mathrm{pA} / \sqrt{\mathrm{Hz}}$, far too large. Increasing the resistor to, e.g., $15 \mathrm{k} \Omega$ doesn't work either, because then the bandwidth will be only $700 \mathrm{kHz}$ due to the photodiode capacitance (assumed to be $15 \mathrm{pF}$, typical for a small InGaAs diode).
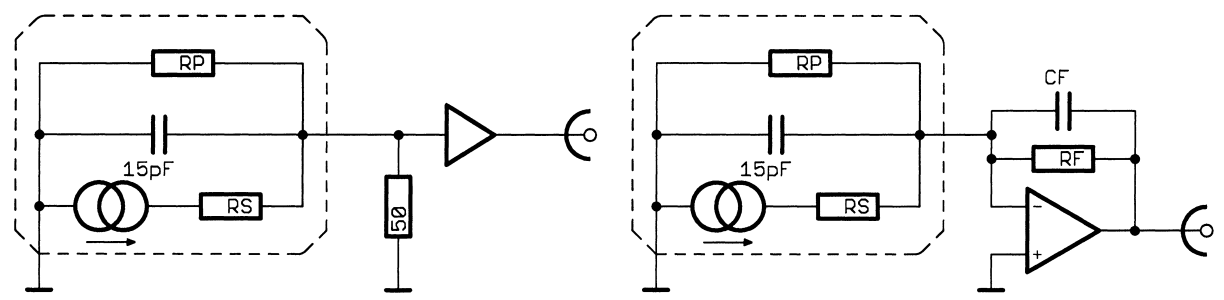

FIGURE 3. Two standard methods for a photodiode preamplifier. The components in the dashed frame represent the equivalent circuit of the photodiode.

The right part of Figure 3 shows a standard transimpedance amplifier using an opamp. The op-amp would need a bandwidth of $f_{T}>(20 \mathrm{MHz})^{2} / 700 \mathrm{kHz} \approx 600 \mathrm{MHz}[6]$ and a current noise of $\widetilde{I_{n}}<1 \mathrm{pA} / \sqrt{\mathrm{Hz}}$. No such op-amp exists. But even if we had an op-amp available with e.g. $f_{T}=1 \mathrm{GHz}, \widetilde{I_{n}}=1 \mathrm{pA} / \sqrt{\mathrm{Hz}}$ and $\widetilde{U_{n}}=1 \mathrm{nV} / \sqrt{\mathrm{Hz}}$, the performance would be marginal.
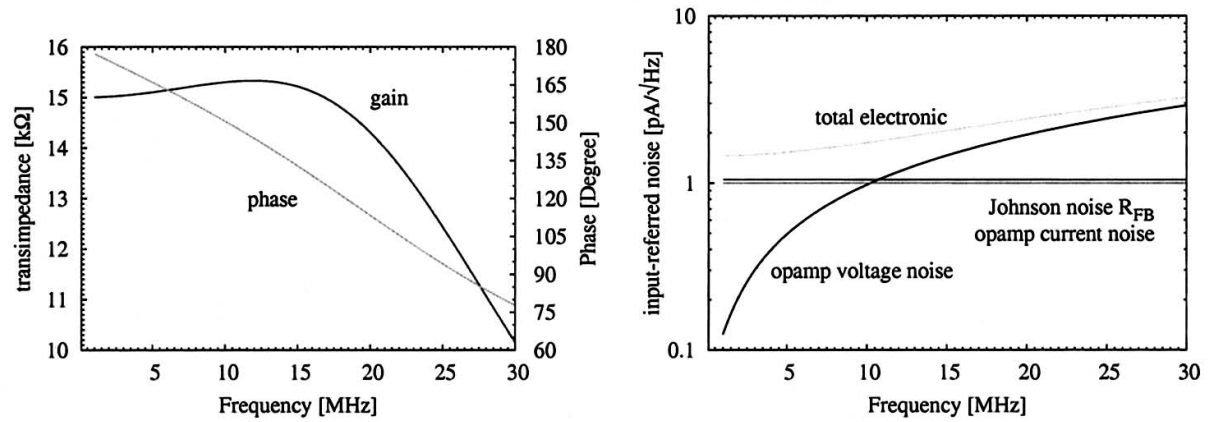

FIGURE 4. Transimpedance amplifier performance with near-ideal opamp. LISO simulation with $\mathrm{R}_{\mathrm{F}}=15 \mathrm{k} \Omega$ and $\mathrm{C}_{\mathrm{F}}=0.5 \mathrm{pF}$. 
Figure 4 shows the simulated transfer function and noise in this case. While the transfer function is acceptable, the noise increases towards higher frequencies, which can be understood by considering the noise gain that amplifies the input voltage noise $\widetilde{U}_{\mathrm{n}}$ of the amplifier (Figure 5):

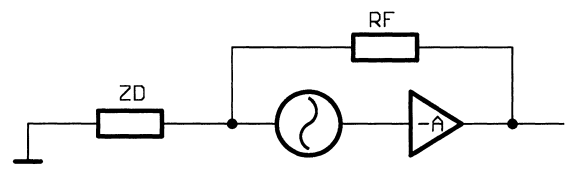

FIGURE 5. Noise gain in transimpedance amplifier.

For high gain $|A| \gg 1$, we have at the output: $\widetilde{U}_{\text {out }}=\widetilde{U}_{\mathrm{n}}\left(1+R_{\mathrm{F}} /\left|Z_{\mathrm{D}}\right|\right) \approx$ $\widetilde{U}_{\mathrm{n}}\left(1+2 \pi f R_{\mathrm{F}} C_{\mathrm{D}}\right)$. Referred to the input: $\widetilde{I}=\widetilde{U}_{\text {out }} / R_{\mathrm{F}}=\widetilde{U}_{\mathrm{n}} / R_{\mathrm{F}}+\widetilde{U}_{\mathrm{n}} 2 \pi f C_{\mathrm{D}}$. At $20 \mathrm{MHz}, 15 \mathrm{pF}$ yields $\left|Z_{\mathrm{D}}\right|=530 \Omega$, and even an excellent $\widetilde{U}_{\mathrm{n}}=1 \mathrm{nV} / \sqrt{\mathrm{Hz}}$ yields an equivalent input noise of $1.9 \mathrm{pA} / \sqrt{\mathrm{Hz}}$. Changing $R_{\mathrm{F}}$ does not help. So we have to maximize $\left|Z_{\mathrm{D}}\right|$, e.g. by minimizing $C_{\mathrm{D}}$.

We are now investigating better amplifiers (built from discrete $\mathrm{GHz}$ transistors) as well as impedance matching circuits to increase the effective photodiode impedance. Preliminary results suggest that an total electronic input noise of $2.5 \mathrm{pA} / \sqrt{\mathrm{Hz}}$ might be achievable, but not $1 \mathrm{pA} / \sqrt{\mathrm{Hz}}$. This would mean that the local oscillator power on PDM may need to be increased to e.g. $300 \ldots 1000 \mu \mathrm{W}$ per quadrant, depending on the budget allocation. The task gets easier with smaller photodiode capacitance.

\section{VOLTAGE REFERENCES}

Another component whose importance for the performance of LISA has recently been recognized are voltage references, which are needed in several places:

- Laser power stabilization,

- Piezo drivers for the point-ahead actuator and reference cavity,

- Photodiode bias,

- Capacitive actuators of the test mass.

Manufacturers usually do not specify the noise at $\mathrm{mHz}$ frequencies, and never as spectral density. We developed a measurement setup to characterize the long-term stability (Figure 6).

By comparing two identical, but independent references against each other, the measurement yields $\sqrt{2}$ times the noise of one reference, if both references have the same amount of uncorrelated noise. The additional noise of a subtracting circuit is avoided by using an independent floating voltage supply for one of the references and connecting their 'hot' ends such that the voltage difference develops near ground and can directly be amplified with an auto-zero ('chopper') amplifier such as AD8628.

We have thus measured a few selected $5 \mathrm{~V}$ and $10 \mathrm{~V}$ references from Analog Devices, Linear Technology and Maxim in their best available grades. Figure 7 shows the results in normal laboratory conditions (open PCB, no temperature stabilization). 


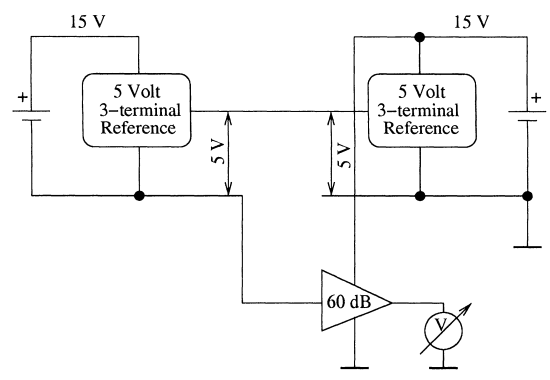

FIGURE 6. Measurement setup for voltage reference characterization.
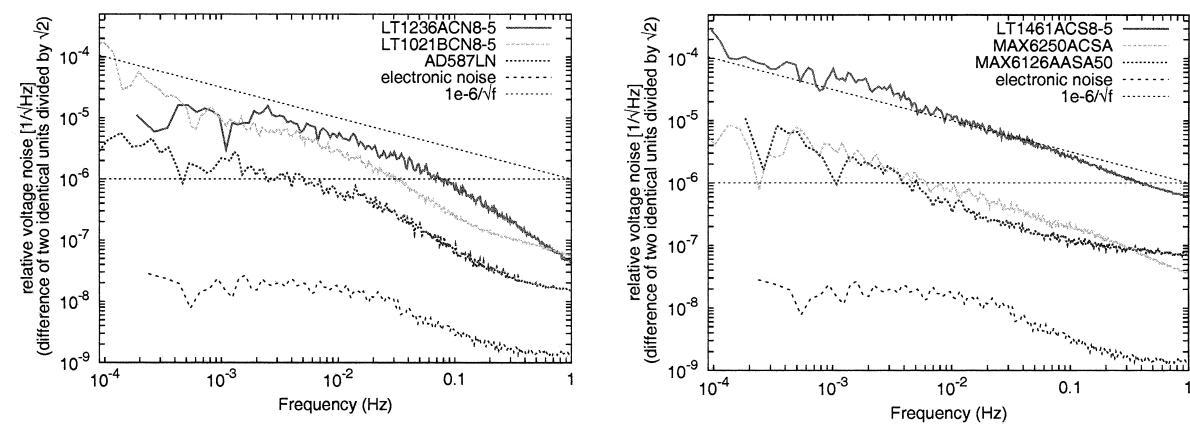

FIGURE 7. Measured voltage noise of selected $5 \mathrm{~V}$ references without temperature stabilization. Left: DIP case, right:SMD case.

To further improve the low-frequency performance, the temperature of the IC was actively stabilized using an NTC sensor and a Peltier element in a small aluminum block that enclosed the reference IC. For practical reasons, we have implemented this stabilization only for those reference ICs with a DIP case so far. The loop gain was not yet optimized, and the resulting stability is worse than what is expected in orbit. The results are shown in Figure 8. The AD587LN with temperature stabilization almost reaches $10^{-6} / \sqrt{\mathrm{Hz}}$ down to $0.1 \mathrm{mHz}$, which approximates the level required for the recently proposed scheme of DC actuation on the test mass along the sensitive axis[7].

In an attempt to further reduce the low-frequency noise, we tried to use an active filter with a corner frequency of $1 \mathrm{mHz}$, an auto-zero amplifier and power supplies bootstrapped from the stabilized output (Figure 9). Unfortunately, the noise of this circuit was much higher than the previous results. The reason is that in any low-pass filter, a DC voltage needs to be across at least one capacitor. The temperature coefficient of largevalue capacitors is $\mathrm{d} C / \mathrm{d} T>100 \mathrm{ppm} / \mathrm{K}$, more typically $300 \mathrm{ppm} / \mathrm{K}$, much higher than the temperature coefficient of the reference IC itself. Since the charge on the capacitor is momentarily conserved, any temperature fluctuation $\mathrm{d} T$ translates into a voltage fluctuation with the same coefficient, which completely spoils the reference $\mathrm{d} V / \mathrm{d} T$ of $<5 \mathrm{ppm} / \mathrm{K}$. Hence we have to rely on the stability and low noise of the reference itself. 


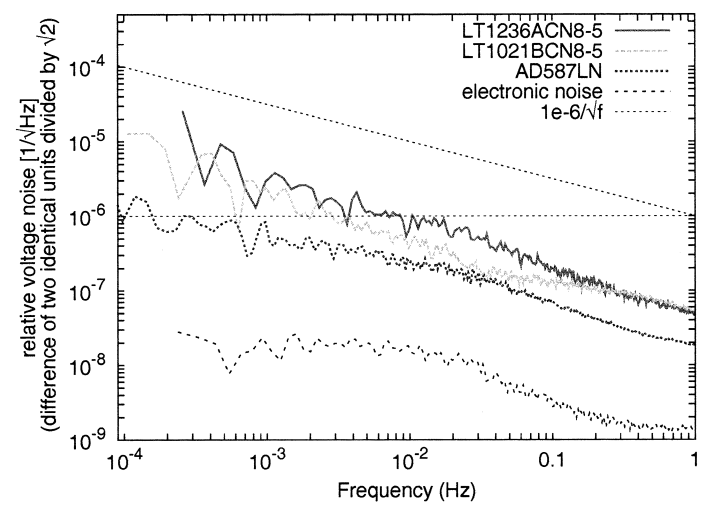

FIGURE 8. References in DIP case with moderate temperature stabilization.

Further experiments on this subject are ongoing.

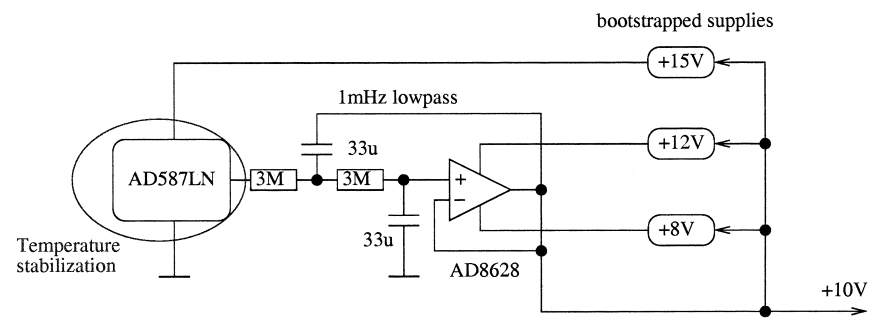

FIGURE 9. Failed attempt to improve stability by filtering.

\section{ACKNOWLEDGMENTS}

We acknowledge support from the DLR for our experiments.

\section{REFERENCES}

1. A Povoleri et al, This Volume pp. ?-?

2. D Weise et al, This Volume pp. ?-?

3. P Gath et al, This Volume pp. ?-?

4. G Heinzel et al, Class. Quant. Grav. 23 pp. S119-S124

5. H Schulte et al, This Volume pp. ?-?

6. The bandwidth of a transimpedance amplifier is approximately $\sqrt{f_{1} \times f_{2}}$ where $f_{1}$ is the bandwidth of the op-amp and $f_{2}=1 /\left(2 \pi R_{\mathrm{F}} C_{\mathrm{D}}\right)$ with the feedback resistor $R_{\mathrm{F}}$ and the source capacitance $C_{\mathrm{D}}$.

7. P Bender, "Cancellation of differential accelerations for the LISA spacecraft", Class. Quant. Grav., article in press, expected publication: October 2006 